Longitudinal Motion Near Unstable Fixed Point

J. Wei and S. Y. Lee

$B N L$

September 12, 1988 


\title{
LONGITUDINAL MOTION NEAR UNSTABLE FIXED POINT
}

\author{
J. Wei and S. Y. Lee \\ Accelerator Development Department \\ Brookhaven National Laboratory \\ Upton, New York 11973
}

\begin{abstract}
The longitudinal motion of a bunched particle beam near the unstable fixed point is studied analytically. Numerical tracking is used to simulate the evolution of a contour of a certain phase space area under the bunch rotation process, where the unstable fixed point is used to develop a mismatched distribution before the rotation. Based on the proposed beam transfer from the Brookhaven National Laboratory AGS to RHIC, criteria on rotation timing and bunch area growth induced by the inaccuracy in synchronous phase angle shifting are discussed.
\end{abstract}




\section{INTRODUCTION}

When a bunched beam is transfered from one R.F. system of the accelerator to the other, an important consideration is the beam dilution in the longitudinal phase space caused by bunch shape mismatch and non-linear motion. To match the bunch shape, R.F. voltage may turn out to be outside of the tuning range of the R.F. system. Bunch rotation techniques sometimes provide solution.

The AGS at the Brookhaven National Laboratory serves as the injector for the proposed Relativistic Heavy Ion Collider (RHIC). The existing R.F. system of the AGS operates at a peak voltage of $300 \mathrm{kV}$, with a frequency range of $2.5 \sim 4.46 \mathrm{MHz}$. The proposed acceleration R.F. system of RHIC has a frequency of $26.7 \mathrm{MHz}$. A bunch rotation on the AGS before extraction is very helpful in achieving a bunch of the beam which is matched to the acceleration R.F. bucket of RHIC. Since the peak voltage of the AGS R.F. system may not be conveniently adjusted, the method of using unstable fixed point to provide a mismatched bunch for rotation becomes interesting.

For the proton operation in RHIC, the injection energy is above the transition energy. Bunch rotation gymnastic discussed in this section can be used to inject the beam directly into the high frequency R.F. system $(160 \mathrm{MHz})$ of RHIC. In this scenario, the bunch length should be shortened by a factor of 4 to fit into the high frequency bucket.

In section II of this paper, we shall study the longitudinal motion of a particle distribution near the unstable fixed point. The simulation of the bunch rotation process on the AGS is discussed in section III.

\section{LONGITUDINAL MOTION NEAR UNSTABLE FIXED POINT}

The longitudinal motion of a particle of electric charge of $Z$, atomic mass number of $A$ can be described by the differential equations

$$
\frac{d}{d t}\left(\frac{\Delta E}{\Omega_{0}}\right)=\frac{Z e \hat{V}}{2 \pi} \cdot\left(\sin \phi-\sin \phi_{s}\right)
$$




$$
\frac{d}{d t}\left(\phi-\phi_{s}\right)=\frac{h \Omega_{0}^{2} \eta}{\beta^{2} E} \cdot \frac{\Delta E}{\Omega_{0}}
$$

where a sinosoidal R.F. voltage has been assumed, and

$E=A m_{0} c^{2} \gamma$ synchronous energy of the particle

$\gamma=\left(1-\beta^{2}\right)^{-\frac{1}{2}}$ synchronous energy of the particle in $m_{0} c^{2}$ units

$\eta=\frac{1}{\gamma_{t}^{2}}-\frac{1}{\gamma^{2}}$

$\phi=$ the particle R.F. phase

$\Delta E=$ deviation of the particle energy from the synchronous energy

$h=$ R.F. harmonic number.

When the bunch area is small in comparison with the bucket area, equations (1) and (2) can be conveniently approximated by the linearization. Near the unstable fixed point,

$$
\begin{gathered}
\dot{\delta}=-C_{1} \cdot \Delta \phi \\
\dot{\Delta \phi}=C_{2} \cdot \delta,
\end{gathered}
$$

where $\delta=\frac{\Delta p}{p}$ is the relative deviation of the particle momentum, $\Delta \phi=\phi-\left(\pi-\phi_{s}\right)$ and

$$
C_{1}=\frac{Z e \hat{V} \Omega_{s} \cos \phi_{s}}{2 \pi A m_{0} c^{2} \gamma}, C_{2}=2 \pi h \eta \Omega_{0} .
$$

A particle of coordinates $\left(\delta_{0}, \phi_{0}\right)$ at an initial time of $t=0$ followes the hyperbolic invariant contour

$$
-\frac{\delta^{2}}{\hat{\delta}^{2}}+\frac{\phi^{2}}{\hat{\phi}^{2}}=1
$$

with

$$
\hat{\delta}^{2}=\frac{\Omega_{s}^{2}}{C_{2}^{2}} \phi_{0}^{2}-\delta_{0}^{2}, \hat{\phi}^{2}=\phi_{0}^{2}-\frac{C_{2}^{2}}{\Omega_{s}^{2}} \delta_{0}^{2},
$$

where $\Omega_{s}=-C_{1} C_{2}$ is the linear synchrotron oscillation frequency.

At a time $t=t_{1}$ the new coordinates in the longitudinal phase space are

$$
\begin{gathered}
\delta=\frac{\Omega_{s}}{C_{2}}\left(-\frac{1}{2}\left(\phi_{0}-\frac{C_{2}}{\Omega_{s}} \delta_{0}\right) \exp \left(-\Omega_{s} t\right)+\frac{1}{2}\left(\phi_{0}+\frac{C_{2}}{\Omega_{s}} \delta_{0}\right) \exp \left(\Omega_{s} t\right)\right) \\
\phi=\frac{1}{2}\left(\phi_{0}-\frac{C_{2}}{\Omega_{s}} \delta_{0}\right) \exp \left(-\Omega_{s} t\right)+\frac{1}{2}\left(\phi_{0}+\frac{C_{2}}{\Omega_{s}} \delta_{0}\right) \exp \left(\Omega_{s} t\right) .
\end{gathered}
$$


On the other hand, a particle near the stable fixed point $\phi_{s}$ followes the elliptic invariant contour of

$$
\frac{\delta^{2}}{\hat{\delta}^{2}}+\frac{\phi^{2}}{\hat{\phi}^{2}}=1
$$

Imaging that at time $t=0$ the synchronous phase angle is shifted from $\phi_{s}$ to $\pi-\phi_{s}$, at $t>0$ this contour is tilted and gradually, exponentially elongated as

$$
\frac{1}{2}\left(\phi-\frac{C_{2}}{\Omega_{s}} \delta\right)^{2} \exp \left(2 \Omega_{s} t\right)+\frac{1}{2}\left(\phi+\frac{C_{2}}{\Omega_{s}} \delta\right)^{2} \exp \left(-2 \Omega_{s} t\right)=\phi_{0}^{2}-\frac{C_{2}^{2}}{\Omega_{s}^{2}} \delta_{0},
$$

or,

$$
\frac{1}{\left(\phi_{0}^{2}-\frac{C_{2}}{n_{s}} \delta_{0}\right)} \cdot\left[\cosh \left(2 \Omega_{s} t\right) \phi^{2}+\frac{C_{2}^{2}}{\Omega_{s}^{2}} \cosh \left(2 \Omega_{s} t\right) \delta^{2}-\frac{2 C_{2}}{\Omega_{s}} \sinh \left(2 \Omega_{s} t\right) \delta \phi\right]=1 .
$$

We assume that at time $t=t_{1}$ the synchronous phase is shifted back to $\phi_{s}$. At time $t_{2}=t_{1}+\frac{3}{8} \tau_{s}$, where $\tau_{s} \equiv \frac{2 \pi}{n_{s}}$, the contour is rotated along the momentum axis of the longitudinal phace space. At this particular moment the contour is simply described by

$$
\frac{\delta^{2}}{\hat{\delta}_{2}^{2}}+\frac{\phi^{2}}{\hat{\phi}_{2}^{2}}=1
$$

in which $\hat{\delta}_{2}$ and $\hat{\phi}_{2}$ is expressed by their coorresponding quantities at $t=0$,

$$
\hat{\delta}_{2}=\hat{\delta} \cdot \exp \left(\Omega_{s} t_{1}\right), \quad \hat{\phi}_{2}=\hat{\phi} \cdot \exp \left(-\Omega_{s} t_{1}\right)
$$

The momentum spread is thus increased by a factor of $f=\exp \left(\Omega_{s} t_{1}\right)$.

In the case that the synchronous phase is shifted with an error $\Delta$, i.e. from $\phi_{s}$ to $\pi-\phi_{s}+\Delta$, the center of the elliptic contour is displaced both in the momentum and in the phase direction upon the completion of the rotation process. The contour at $t=t_{2}$ becomes then,

$$
\frac{\left(\delta+\Delta_{\delta}\right)^{2}}{\hat{\delta}_{2}^{2}}+\frac{\left(\phi+\Delta_{\phi}\right)^{2}}{\hat{\phi}_{2}^{2}}=1
$$

where

$$
\begin{aligned}
\Delta_{\delta} & =\Delta \cdot \frac{\Omega_{s}}{C_{2}} \exp \left(\Omega_{s} t_{1}\right) \\
\Delta_{\phi} & =\Delta \cdot \exp \left(-\Omega_{s} t_{1}\right)
\end{aligned}
$$


We observe that the phase error $\Delta$ gives an equal amount of relative displacement in either direction

$$
\frac{\Delta_{\delta}}{\hat{\delta}_{2}}=\frac{\Delta_{\phi}}{\hat{\phi}_{2}}=\frac{\Delta \cdot \exp \left(-\Omega_{s} t_{1}\right)}{\hat{\delta}_{2}} .
$$

\section{BUNCH ROTATION ON THE AGS}

Bunch rotation technique offers a convenient way of manipulating the distribution of the bunched beam in the longitudinal phase space, provided that the beam occupies relatively small amount of the R.F. bucket and that the process can be accomplished within a fraction of the synchrotron oscillation period. Using unstable fixed point to achieve the desired distribution before rotation has the advantage that the R.F. peak voltage does not have to be changed during the entire rotation process.

Fig.1a shows a contour which encloses $0.3 \mathrm{eV} \cdot \mathrm{sec}$ proton beam. The rotation is performed at the extraction energy of $\gamma=30$. The R.F. peak voltage remains at $300 \mathrm{kV}$. The synchrotron oscillation period is $\tau_{s}=5 \mathrm{~ms}$. The beam "sits" on the unstable fixed point for a time of $t_{1}=\frac{\ln 2}{2 \pi} \tau_{s}$, as shown in Fig.1b. The beam is expected to be extracted at the time $t_{2}=t_{1}+\frac{3}{8} \tau_{s}$. The beam doubles its momentum spread. The bunch is matched to a bucket which has an effective peak voltage of $2^{4}$ as high as the original one. Fig.2 shows the similar diagrams as Fig.1, with a $t_{1}=\frac{\ln 4}{2 \pi} \tau_{s}$. Due to the nonlinear R.F. voltage, the beam contour starts to deform from its elliptical shape. The deformation is related to the maximum R.F. voltage of $300 \mathrm{kV}$. At the R.F. voltage of $300 \mathrm{kV}$, an increase in the momentum spread by a factor of 4 , or equivalently a decrease in the bunch length by a factor of 4 results in a small nonlinear effect. At a higher voltage, the tolerable compression factor becomes larger. In Fig. 3 the requested bunch length compression factor, $f=\exp \left(\Omega_{s} t_{1}\right)$, is plotted as a function of the time $t_{1}$.

An inaccuracy in the shifting of the synchronous phase angle may result in an effective bunch area growth. In Fig.4 we assume that this error is $\Delta=4.5^{\circ}$ and that $f=2$. The 
estimated dilution in the longitudinal phase space is

$$
\frac{\varepsilon_{2}}{\varepsilon}=\frac{\hat{\delta}+\Delta_{\delta}}{\hat{\delta}} \cdot \frac{\hat{\phi}+\Delta_{\phi}}{\hat{\phi}} \sim 2
$$

The displacement of the center of the bunch in momentum direction and the effective bunch area increment are plotted against the phase shifting error in Fig.5. 


\section{FIGURE CAPTIONS}

Fig. 1. Proton bunch rotation on the AGS using the unstable fixed point. The bunch length compression factor is $f=2$.

Fig. 2. Proton bunch rotation on the AGS using the unstable fixed point. The bunch length compression factor is $f=4$.

Fig. 3. The requested bunch length compression factor $f$ as a function of the time for the beam to stay on the unstable fixed point .

Fig. 4. Proton bunch rotation on the AGS using the unstable fixed point. The bunch length compression factor is $f=2$. The synchronous phase is shifted with an error $\Delta=4.5^{\circ}$.

Fig. 5. The displacement of the center of the bunch in momentum axis and the effective bunch area increase as a function of the phase shifting error $\Delta$. 

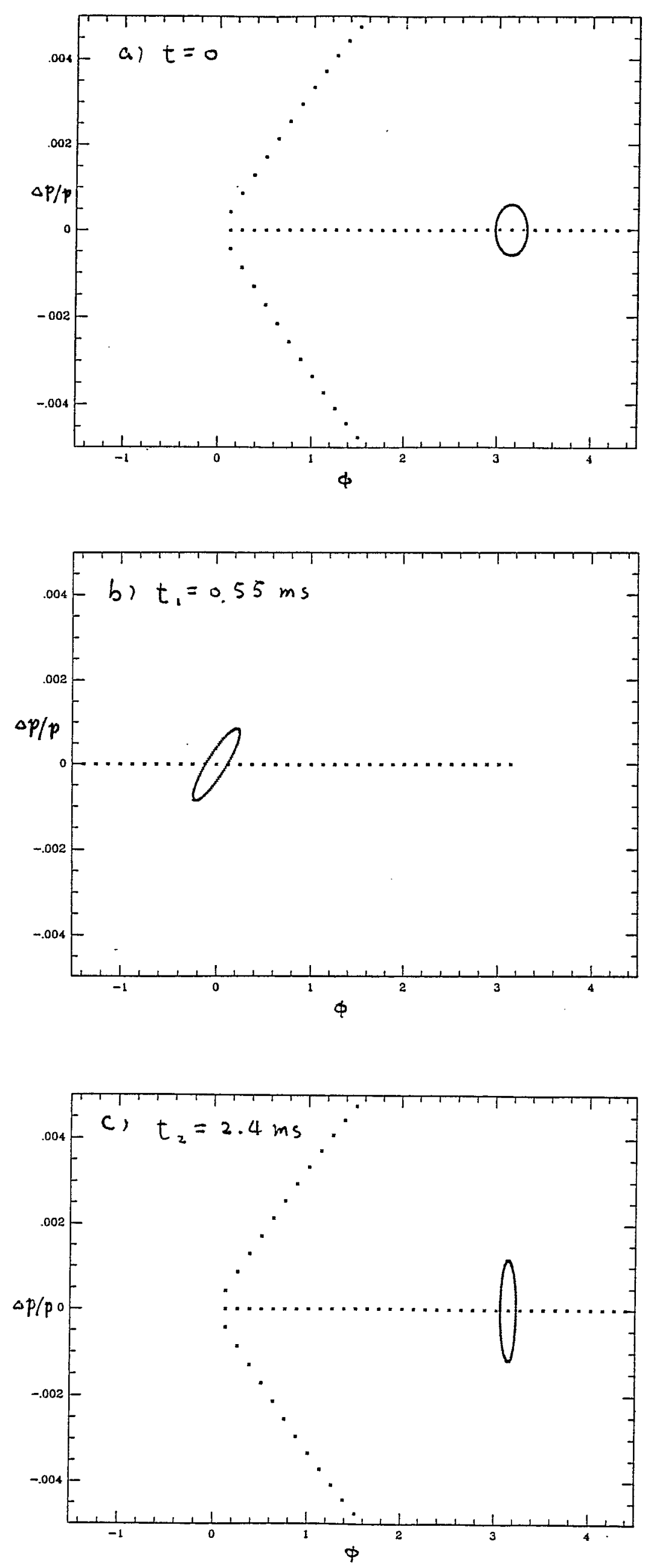

Fig. 1 

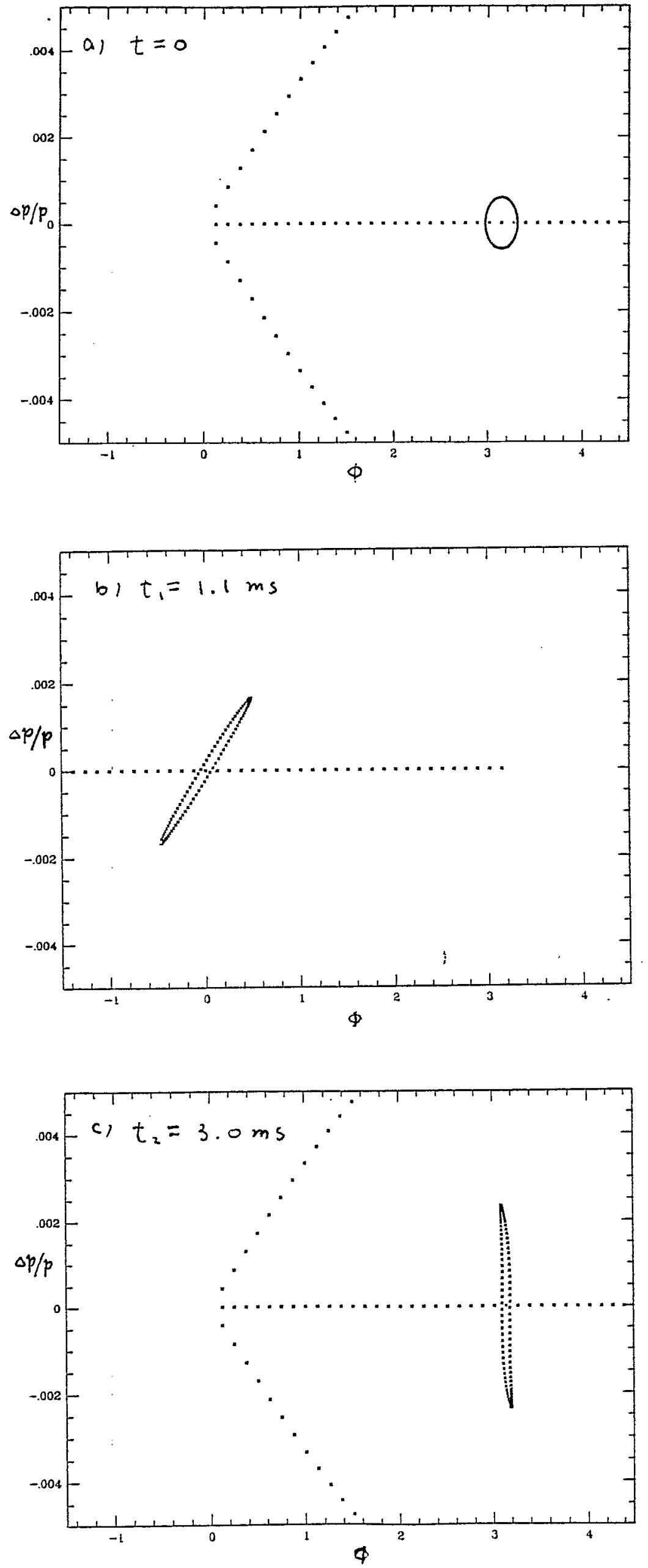

Fig. 2 


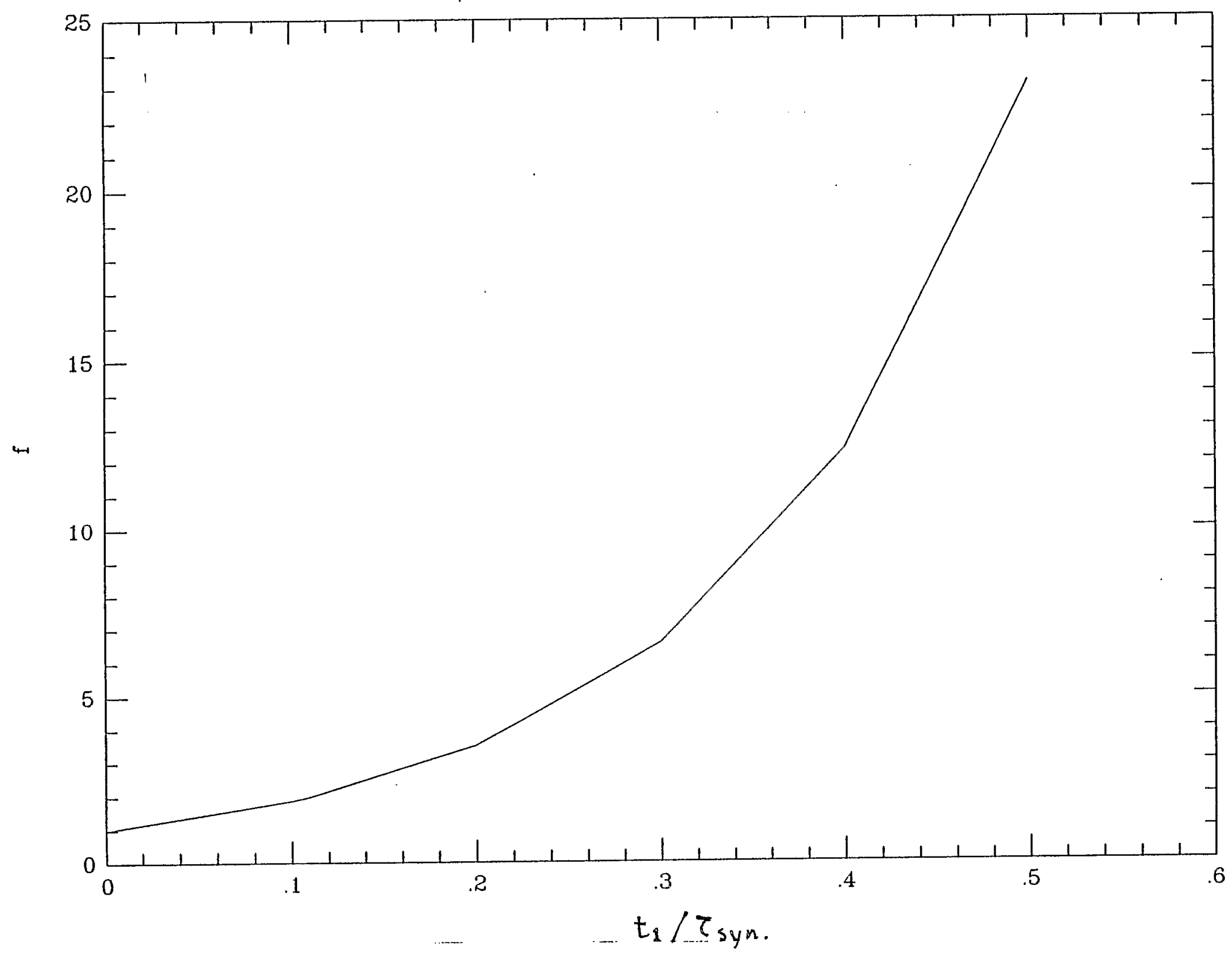

Fig. 3 

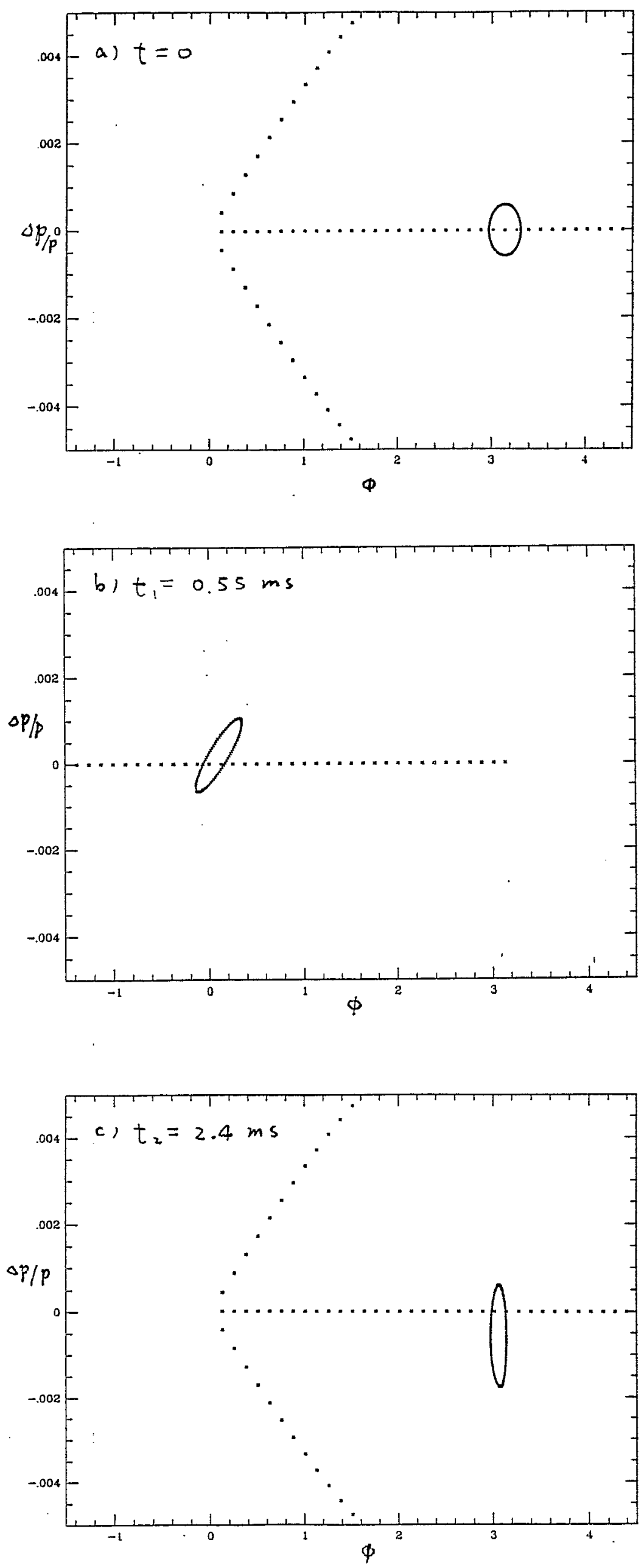

Fig. 4 

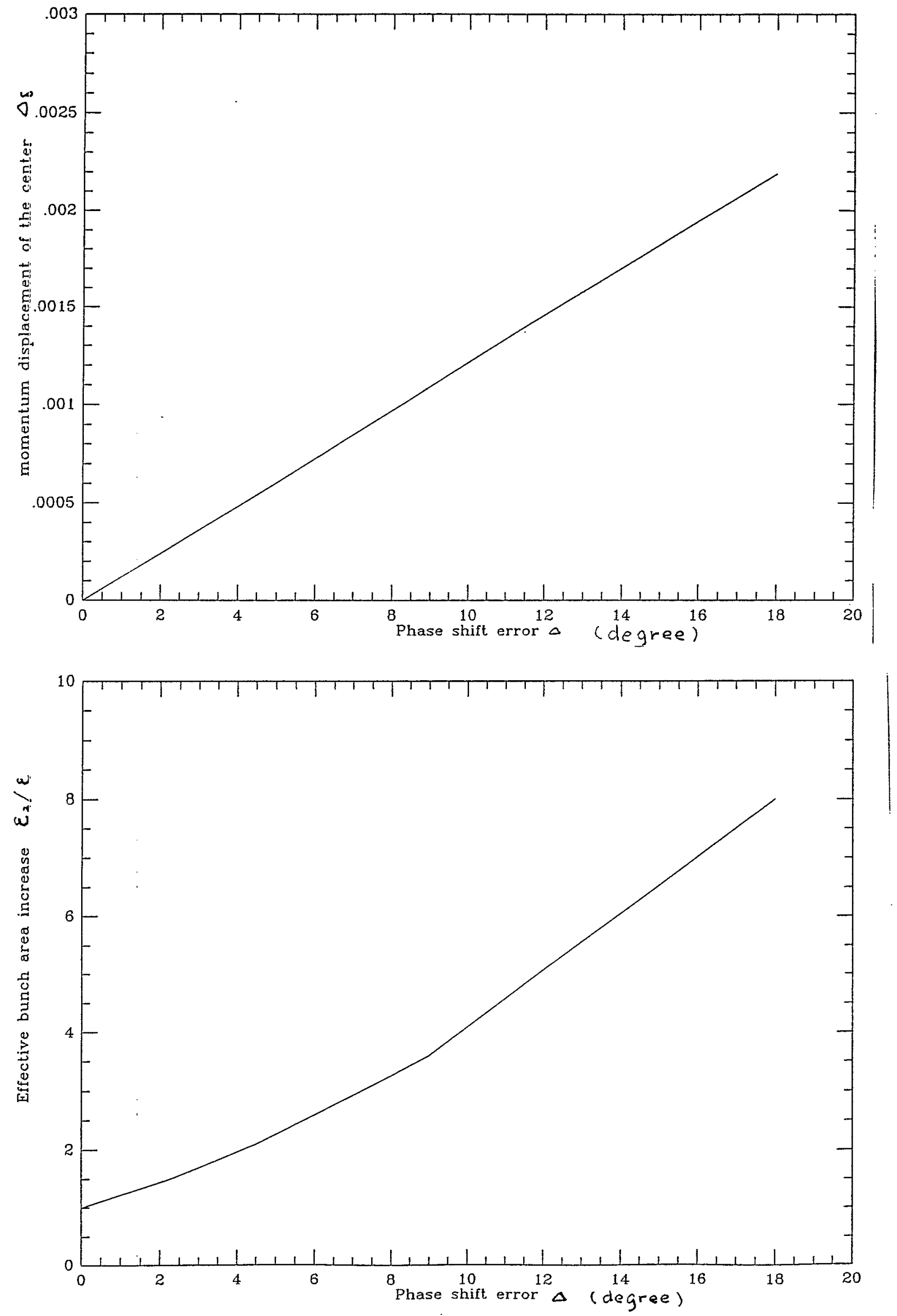

Fig. 5 